\title{
The beneficial effects of tree nuts on the aging brain
}

\author{
Amanda N. Carey, Shibu M. Poulose and Barbara Shukitt-Hale* \\ United States Department of Agriculture, Agricultural Research Services, Human Nutrition Research \\ Center on Aging, Tufts University, Boston, MA, USA
}

\begin{abstract}
Dietary patterns may play an important role in protecting the brain from the cellular and cognitive dysfunction associated with the aging process and neurodegenerative diseases. Tree nuts are showing promise as possible dietary interventions for age-related brain dysfunction. Tree nuts are an important source of essential nutrients, like vitamin E, folate, and fiber. Tree nuts also contain a variety of components, such as phytochemicals like flavonoids, proanthocyanidins, and phenolic acids, as well as monounsaturated and omega- 3 and omega- 6 polyunsaturated fatty acids that have the potential to combat age-related brain dysfunction. Evidence is accumulating that suggests that tree nuts and their bioactive constituents have the potential to reduce oxidative stress and inflammation, as indicated by decreased lipid peroxidation in vivo and reduced production of the free radical nitric oxide and the pro-inflammatory cytokine tumor necrosis factor-alpha in vitro. Also, tree nut consumption might have the ability to mitigate some of the cognitive decline associated with aging. Here we review the current knowledge of how the consumption of nuts may improve brain health, specifically focusing on walnuts, almonds, pistachios, and pecans.
\end{abstract}

Keywords: Brain, aging, walnut, nut, polyunsaturated fatty acids, antioxidant effects

\section{Introduction}

In the brain, aging has been characterized by losses in neuronal function accompanied by behavioral declines such as decrements in motor and cognitive performance, in both animals and humans. Oxidative stress and inflammation are thought to be important factors in brain aging and age-related neuronal dysfunction [1,2]. The central nervous system, particularly during aging, is highly susceptible to oxidative stress and inflammation, with both increasing in tandem with age, compiled with a loss of efficiency by which the body's natural defense mechanisms mitigate these insults. In essence, aging is a condition where stressors are not countered by protective functions, leading to a cascade of altered signaling and ultimately to the injury or death of neurons, resulting in permanent modification of biological and behavioral processes, increasing the risk of various neurodegenerative

${ }^{*}$ Corresponding author: Barbara Shukitt-Hale, Neuroscience and Aging Lab, USDA HNRCA at Tufts University, 711 Washington Street, Boston, MA 02111, USA. Tel.: +1 617556 3118; Fax: +1 617556 3222; E-mail: barbara.shukitthale@ars.usda.gov. diseases, and immensely compromising the quality of life [3-6]. Apart from being debilitating in nature on the elderly, these diseases are an enormous economic burden on society with a direct annual economic cost for caregiving in Alzheimer's disease alone estimated at $\$ 183$ billion in 2011 [7]. However, it is worth noting that the clinical manifestation of these devastating diseases takes approximately 8-10 years, and in certain cases up to 25 years, indicating a covert initiation and progression originating in the brain. In the search for a cure for these age-related diseases, prevention has received much less attention; however, a growing number of studies suggest the potential benefit of boosting the brain's endogenous defenses by supplementing with fruits and vegetables rich in natural phytochemicals.

Evidence is accumulating to suggest that dietary patterns may play an important role in protecting the aging brain from cellular and cognitive dysfunction. Dietary supplementation with a number of foods has been shown to induce a multiplicity of protective effects against some aspects of age-related behavioral and cellular dysfunction [8]. Higher consumptions of certain foods or specific dietary patterns, such as the 
Mediterranean diet, are associated with reduced risk of cognitive impairment, which is thought to be due to the increase in nutrient intake [9-11]. For example, in a prospective cohort study, Gu and colleagues [12] found that, in people 65 years of age or older, a dietary pattern of eating salad dressing, nuts, fish, tomatoes, poultry, cruciferous vegetables, dark and green leafy vegetables, and fruits, while low in high-fat dairy, red meat, organ meat, and butter consumption, is significantly associated with Alzheimer's disease risk and is strongly protective against its development. This dietary pattern reflects a diet rich in omega- 3 and omega- 6 polyunsaturated fatty acids (PUFAs), vitamin E, and folate and poor in saturated fat and vitamin B12.

One of the food categories showing promise as a possible dietary intervention for age-related brain dysfunction is tree nuts, which are important sources of nutrients for humans that have been used by ancient civilizations to prevent or treat disease [13]. Currently, the dietary recommendations established by the United States Department of Agriculture states that tree nuts are an important source of dietary protein, with half an ounce of nuts (approximately 14 grams) considered a daily serving of protein, and are also important sources of dietary fiber and unsaturated fats [14]. In contrast to the botanical classification, the common culinary and English usage of the term "nut" may refer to many hardened edible kernels (i.e. seed) referred to as "nuts". In the United States, tree nuts such as almonds (Prunus dulcis), cashews (Anacardium occidentale), macadamias (Macadamia integrifolia and tetraphylla), pecans (Carya illinoinensis), pistachios (Pistacia vera), and walnuts (Juglans regia) are commonly consumed. Among these, almonds, walnuts, pistachios, and pecans are the major tree nuts that are domestically grown and constitute more than $75 \%$ of the total consumed among all tree nuts utilized in the United States (Table 1). Most current scientific research on the health benefits of nuts is from the field of cardiovascular research [15, 16], and a limited amount of research is focused on the health benefits of nuts for the brain. The Adventist Health Study [17], a prospective cohort study comparing Seventh-Day Adventists who often maintain a vegetarian or vegan diet versus non-Adventists, found that those who consumed nuts more than four times a week experienced fewer fatal coronary heart disease events compared to those who consumed nuts less than one time per week. Along these lines, the cross-sectional Nurses' Health Study [18] found that women who frequently con-
Table 1

Production and utilization of major tree nuts in the US marketplace in 2010-2011 season [108]

\begin{tabular}{lccr}
\hline Tree nuts & $\begin{array}{c}\text { Total production } \\
(\mathrm{lbs})\end{array}$ & $\begin{array}{c}\text { Domestic } \\
\text { utilization }(\mathrm{lbs})\end{array}$ & \multicolumn{1}{c}{$\begin{array}{c}\text { Exports } \\
(\mathrm{lbs})\end{array}$} \\
\hline Almonds & $1,612,084$ & 499,353 & $1,188,239$ \\
Walnuts & 436,785 & 216,000 & 301,665 \\
Pistachios & 250,125 & 169,404 & 79,578 \\
Pecans & 140,407 & 167,432 & 54,405 \\
All tree nuts & $2,478,896$ & $1,315,840$ & $1,711,460$ \\
\hline
\end{tabular}

sumed nuts had a reduced risk of fatal coronary heart disease and non-fatal myocardial infarction. Moreover, prospective data in US male physicians suggests that men who consumed nuts two or more times per week had reduced risks of sudden cardiac death and total coronary heart disease death compared to men who rarely or never consumed nuts [16]. Given the potent anti-inflammatory effects that nut consumption can have in the cardiovascular system, it stands to reason that nuts may also act as anti-inflammatory agents in the brain. Nuts contain a variety of components, such as phytochemicals and PUFAs that may have the potential to combat age-related brain dysfunction. Here we review the current knowledge of how the consumption of nuts may improve brain health.

\section{Polyunsaturated fatty acids from tree nuts: Potential health benefits}

Although every nut has different combinations and levels of various nutritional components, nuts are known to be good sources of fat, protein [19], and phytosterols [20]. However, most of the tree nuts vary widely in their fatty acid composition (Table 2) and therefore might contribute differentially to health benefits. It should be noted that, as nuts are high in fat and therefore energy-dense, consumers sometimes regard them as fattening foods. However, data suggest that adding nuts to a diet generally does not produce weight gain and may even help with weight loss [21]. Furthermore, nuts are most known and touted for their richness in unsaturated fat, which is generally considered to be a healthier type of fat compared to saturated fat [22]. The PUFAs that nuts contain have garnered the most attention. Alpha-linolenic acid (C18:3n-3, ALA) is an omega- 3 fatty acid found in nuts and is the precursor for the long-chain omega-3 fatty acid eicosapentaenoic acid (C20:5n-3, EPA), which is then converted to 
docosahexaenoic acid (C22:6n-3, DHA). The conversion of ALA to EPA and then to DHA involves further desaturation of the fatty acid chain by increasing the number of double bonds and elongation through the addition of carbon atoms. Omega-3 fatty acids have been shown to be anti-inflammatory and to decrease reactive oxygen species production [23]. Linoleic acid (C18:2n-6, LA) is an omega-6 fatty acid found in nuts and is the precursor to arachidonic acid (C20:4n-6, AA). The PUFAs ALA and LA are essential nutrients, as they cannot be synthesized in the body and must be obtained from the diet. They are critical in the formation, stability, and fluidity of neuronal cell membranes, and abnormalities of cell membrane PUFA composition can alter membrane microstructure and result in abnormal signal transduction [24, 25]. Foods high in PUFAs have been shown to protect the brain from numerous insults associated with aging [26-30] and may possibly allay age-related cognitive decline [27, 31-33]. Dietary supplementation with PUFAs has been shown to slow age-related cognitive decline in humans. In a population-based, prospective study in non-demented elderly subjects (65-84 years), it was found that high monounsaturated fatty acid and PUFA energy intakes were significantly associated with better cognitive performance on the Mini-Mental State Examination in a 8.5-year follow-up assessment [34].

The ideal dietary ratio of omega- 6 to omega- 3 has been determined to be somewhere between $5: 1$ and $1: 1$, and may vary with the disease under consideration [35]. However, individuals who consume a Western diet typically eat omega- 6 and omega- 3 fatty acids in a ratio of $15: 1-16.7: 1$, reflective of our over-consumption of omega- 6 PUFAs and underconsumption of omega-3 PUFAs [35]. It should be noted that there are other food sources of PUFAs, such as fish like salmon, sardines, herring, mackerel, and tuna. Fish contain the omega-3 fatty acid DHA. There is debate as to whether it is more beneficial to consume omega-3 PUFAs in the form of DHA rather than ALA for brain health, as DHA, not ALA, is the fatty acid that accumulates in the brain [36]. However, data has shown that ALA is successfully converted to EPA and DHA in the human body [37]. Inconsistencies in data from studies regarding conversion of ALA to EPA and DHA may be due to a number of factors, such as gender, as females demonstrate greater conversion of ALA to EPA and DHA compared to males [37] or genetic variations among people [38]. Most adults in the United States do no consume enough omega-3 fatty acids and it has been suggested that this may be due to an unwillingness or inability to consume marine sources of PUFAs. Nuts may present a pleasant and feasible way to increase the intake of dietary PUFAs, and they can be added to various foods or eaten as a snack. Furthermore, people may be concerned about the mercury content of fish, for example King Mackerel contains $110 \mathrm{mcg} / 4$ ounces [14]. This level of mercury is high enough to be listed as a type of fish that should be avoided by women who are pregnant or breastfeeding. Thus, tree nuts may provide another way to obtain sufficient PUFAs in one's diet if there are concerns about obtaining all of the necessary PUFAs from marine sources.

\section{Health promoting phytochemicals in tree nuts}

Nuts contain an abundance of other nutrients such as vitamin E, fiber, minerals, and folate [39]. Additionally, plants, including nut-bearing plants, synthesize an enormous number of secondary chemical compounds, widely known as phytochemicals, for survival and perpetuity. These include, but are not limited to, flavonoids, carotenoids, phenolic acids, phytosterols, organosulfur compounds, alkaloids, tannins (proanthocyanidins), phenolic aldehydes, and so on [40]. Moreover, tree nuts also vary in their phytochemical composition based on the growing conditions, species or genetic variability, or post-harvest conditions [40]. Major phytochemical contents of the four major tree nuts are given in Table 3 .

Research suggests that phytochemicals extend a multitude of health benefits to humans, mainly through a combination of additive and/or synergistic effects derived from their antioxidant and anti-inflammatory capabilities. Nuts contain a specific class of phytochemicals, phenolic compounds, such as flavonoids, anthocyanidins, phenolic acids, which are potent antioxidants in vitro. These polyphenolics are found in many fruits and vegetables and have been demonstrated to be good candidates for consideration as therapeutics in combating aging and neurodegenerative diseases [41]. As one example, polyphenols have been shown to be involved in the restoration of calcium homeostasis in the striatal and hippocampal regions of the brain, which are crucial for primary and secondary memory functions $[42,43]$. Vinson and Cai [44] measured free and total polyphenols in nine 
Table 2

\begin{tabular}{lcccc}
\multicolumn{4}{c}{ Major fatty acid composition of the four predominantly consumed tree nuts $[109,110]$} \\
\hline Tree nuts & $\begin{array}{c}\text { Total oil } \\
\text { (g/100 g dry nuts })\end{array}$ & \multicolumn{2}{c}{ Major fatty acid composition (\% of total oil) } \\
\cline { 3 - 5 } & & Polyunsaturated & Monounsaturated & Total saturated \\
\hline Almonds & 40.8 & 21.7 & 69.9 & 8.5 \\
Walnuts & 50.8 & 69.0 & 21.2 & 9.5 \\
Pistachios & 51.2 & 30.3 & 58.2 & 9.3 \\
Pecans & 58.8 & 50.3 & 40.6 & 5.6 \\
\hline
\end{tabular}

Table 3

Major phenolics and lipid contents in the leading tree nuts grown in the United States [39, 40, 99]

\begin{tabular}{lcccc}
\hline Tree nuts & $\begin{array}{c}\text { Total phenols } \\
(\mathrm{mg} \mathrm{GAE} / 100 \mathrm{~g})\end{array}$ & $\begin{array}{c}\text { Total flavonoids } \\
(\mathrm{mg} / 100 \mathrm{~g})\end{array}$ & $\begin{array}{c}\text { Proanthocyanidins } \\
(\mathrm{mg} / 100 \mathrm{~g})\end{array}$ & $\begin{array}{c}\text { Phytosterols } \\
(\mathrm{mg} / 100 \mathrm{~g})\end{array}$ \\
\hline Almonds & 418 & 15.25 & 184.02 & 172 \\
Walnuts & 1,556 & 12.74 & 67.25 & 108 \\
Pistachios & 2,016 & 34.01 & 237.34 & 102 \\
Pecans & 1,657 & 18.01 & 494.05 & 214 \\
\hline
\end{tabular}

*GAE; Gallic acid equivalent.

different types of raw and roasted nuts. Walnuts had the greatest amount of free and total polyphenols when combining raw and roasted nut data. It should be noted that much of the phenolics are located in the pellicle of nut, the outer tan brown skin surrounding the kernel of the nut. This thin cover is only $5 \%$ of the nut weight, but it is naturally rich in antioxidant phenolic compounds that help protect the kernel against oxidation (i.e. rancidity) [45]. Thus eating industrial processed nuts that have removed pellicles could decrease much of their antioxidant capacity [46].

\section{Walnuts}

When compared to a variety of tree nuts, such as almonds, cashews, hazelnuts, macadamia, pecans, and pistachios, walnuts (Juglans regia) were found to have the highest total antioxidant capacity as determined by oxygen radical absorbance capacity (ORAC), ferric ion reducing antioxidant power, and trolox equivalent antioxidant capacity assays $[47,48]$. Walnuts are abundant in phenolic compounds, particularly syringic acid and juglone [49]. In a study of walnuts, brazil nuts, pistachios, pecans, and almonds, it was found that roasted and raw walnuts had significantly more free and total polyphenols than all the other raw and roasted nuts, respectively [50]. The study also found that walnut-bound polyphenol antioxidants are twice as effective as those found in almond and five times more effective than peanut in a lipoprotein binding assay that determines the binding and lipoprotein bound antioxidant activity [50].

While many nuts, like almonds and pistachios, contain more monounsaturated fats than PUFAs, walnuts are comprised primarily of PUFAs (Table 2), making them an excellent source of essential dietary PUFAs [51]. Although many of the health benefits of walnuts are attributed to PUFAs, walnuts contain a number of other potential neuroprotective compounds including oleic acid, ellagic acid, vitamin E, folate, and numerous antioxidant polyphenolics [52]. Walnuts also contain melatonin, a hormone synthesized in the pineal gland that is critical for circadian rhythm regulation [53]. Melatonin deficiency has been linked to degeneration of cholinergic neurons in the basal forebrain and deposition of aggregated proteins in the brain, such as amyloid beta peptides, leading to cognitive impairment and dementia [54]. Melatonin is also a free radical scavenger and antioxidant $[55,56]$. Rats that were fasted for 24 hours, and then given ad libitum access to walnuts for four hours, demonstrated a significant increase in serum melatonin levels compared to control rats, which was correlated with an increase in total antioxidant capacity of the serum, indicating the ability of the blood to detoxify free radicals [53].

Also, nuts may reduce inflammation in the cardiovascular system. Walnuts have been shown to be beneficial for cardiovascular health. A walnut- and walnut oil-rich diet reduced the inflammatory and 
cardiovascular risk factors among hypercholesterolemic men and women [57]. These beneficial effects were attributed, at least in part, to reductions in C-reactive protein. These effects were further corroborated in hypercholesterolemic subjects, where walnut diets reduced the production of proinflammatory cytokines in serum [58]. A meta-analysis of the effects of walnuts on lipid parameters found that diets supplemented with walnuts resulted in significant reductions in total and low-density lipoprotein (LDL) cholesterol concentrations, without having an adverse effect on body weight [59]. Reductions in cardiovascular disease risk factors may be associated with better brain health, as cardiovascular disease is also associated with the development of cerebrovascular disease, stroke, and mild cognitive impairment [60, 61].

Even though research reports indicating the specific effects of walnut consumption at the brain cell and molecular level are limited, a few available reports support the beneficial effects of walnuts on brain health. In fact, much of the current research addressing the effects of tree nuts on the brain is from research with walnuts. Walnut oil extract has been shown to inhibit lipopolysaccharide (LPS)-induced activation of microglia cells. Treatment of BV2 microglial cells with methanolic extract of walnut $(0.01,0.1$, and $1 \mathrm{mg} / \mathrm{ml}$ ) prior to LPS exposure, resulted in an attenuation of LPS-induced production of the free radical, nitric oxide, and reduced expression of inducible nitric oxide synthase and the inflammatory cytokine, tumor necrosis factor-alpha [28]. These data suggest that walnuts may be able to mitigate both oxidative stress and inflammation, two purported factors in age-related decline. Further evaluation also revealed that walnut oil extract internalized the LPS receptor, toll-like receptor-4, demonstrating that walnut was having direct effects on microglial cell signaling. Furthermore, Willis and colleagues reported that walnut-supplemented diets enhance cholinergic transmission in the striatum either through increasing acetylcholine (ACh) synthesis or inhibiting $\mathrm{ACh}$ esterase, which hydrolyses $\mathrm{ACh}$, among aged rats [30]. It has been postulated that ACh esterase activity increases with age, leading to dysfunction of cholinergic neurons, in turn leading to cognitive declines similar to Alzheimer's disease [30].

The potential brain health benefits of walnuts were further corroborated by research reporting the effects of walnuts on cognitive behavior in aged rats [33]. In this study, 19 month old rats consumed diets containing
$0 \%, 2 \%, 6 \%$, or $9 \%$ ground English walnut (including skins) for two months. Animals consuming the $2 \%$ and $6 \%$ walnut diet showed some improvement in tests of balance compared to the $0 \%$ walnut-fed group. Furthermore, the rats maintained on a $6 \%$ walnut diet also located a hidden platform sooner in the working memory version of the Morris water maze. However, animals consuming the $9 \%$ walnut diet showed impaired performance on one test of balance and took longer to locate a hidden platform in the Morris water maze during the first of each pair of trials, relative to $0 \%$ walnut fed rats. While too much walnut was disadvantageous, the most beneficial dose is in line with the current FDA recommendations for nut consumption, which is one ounce/day for humans. The brains of these rats were evaluated in a separate study and it was found that the brains of 19 month old rats that were fed a walnut-supplemented diet showed activation of autophagy, a neuronal housekeeping function, and reduced aggregation of polyubiquitinated proteins in the striatum and hippocampus [62]. This activation of neuronal housekeeping function is achieved through inhibiting phosphorylation of the mammalian target of rapamycin, up-regulating ATG5 and Beclin 1 (both ubiquitin-binding proteins), and increasing conversion of MAP1BLC3-I to LC3-II. The clearance of ubiquitinated aggregates such as sequestosome 1 (p62/SQSTM1) was followed by significant reductions in the levels of p38 mitogen activated protein kinase and phosphorylation of nuclear factor kappa B and cyclic AMP response element-binding protein [62].

A recent study in rats further extends the behavioral data in rodents supporting that consumption of walnuts can result in detectable behavioral changes and direct effects on the brain [63]. Rats were orally administered $80 \mathrm{mg}$ of finely crushed walnuts mixed with water, daily for 28 days. As the rats were approximately 200 grams each, this is about $450 \mathrm{mg} / \mathrm{kg} /$ day; in human terms this would be equivalent to a $60 \mathrm{~kg}$ person consuming approximately 27 grams a day. Rats fed the walnut solution demonstrated significantly better memory retention in both elevated-plus and the radial arm mazes compared to rats that did not receive the walnut solution. Walnut consumption also elevated levels of brain serotonin, tryptophan, which is the precursor to serotonin, and 5-hydroxyindoleacetic acid, a metabolite of serotonin, compared to rats not administered the walnut solution. Walnuts are rich in tryptophan, and it is possible that the walnuts increased serotonin metabolism, which may be responsible for 
the increase in memory retention. Previous studies support this hypothesis, as it has been demonstrated that tryptophan administration enhances cognitive performance in rats $[64,65]$. It should also be noted that serotonin imbalance is implicated in anxiety and depression. This may suggest that the results of the elevated-plus maze, which is also a common test of anxiety, may reflect a decrease in anxiety as opposed to or in addition to enhanced memory retention. This is also clinically relevant, as dysregulation of the hypothalamic-pituitary (HPA) axis is common in the aging population and may be associated with an increased risk of developing an anxiety or affective disorder [66, 67]. Drugs that increase serotonin in the brain have been demonstrated to reduce some of the dysfunction associated with HPA axis dysfunction [68], and thus walnuts could offer similar benefits by elevating brain serotonin levels.

Walnuts have also been shown to have the potential to impact Alzheimer's disease. Amyloid-beta (A $\beta$ ) protein is a major component of the senile (i.e. amyloid) plaques that are a hallmark of Alzheimer's disease [69, 70]. $A \beta$ is associated with increases in oxidative stress and death of neurons $[71,72]$. Walnut extract treatment of PC12 cells prevented A $\beta$-induced cell death [73]. The pretreatment of cells with walnut extract also significantly reduced the percentage of DNA fragmentation, which is a marker of apoptosis, induced by $\mathrm{A} \beta$. Furthermore, walnut extract inhibited $A \beta$-induced reactive oxygen species production. Moreover, walnut extract may have anti-amyloidogenic effects. A form of $A \beta$ protein, fibrillar $A \beta$, is the principal component of amyloid plaques [74]. Walnut extract inhibited $A \beta$ fibril formation in a concentration and time-dependent manner and also defibrillized $\mathrm{A} \beta$ that had already been formed [75]. Although these studies were both performed in vitro, these data suggest that a diet rich in walnuts may be able to reduce $A \beta$-mediated toxicity, cell death, and the formation of plaques. An important question, however, is: do the findings discussed here translate into beneficial effects in humans with respect to learning and memory?

In humans, walnuts were found to contribute to increases in brain-derived nerve growth factor (BDNF) [76]. Participants were randomly selected from an ongoing 6 year clinical trial examining the effects of the Mediterranean diet on men and woman aged 55-80 with cardiovascular disease risk factors. Three years into the clinical trial, the participants were assigned to one of the following diets: a control low fat diet,
Mediterranean diet + virgin olive oil, or Mediterranean diet + nuts, and were maintained on these diets for an additional 3 years. The nuts component of the diet consisted of daily intake of $15 \mathrm{~g}$ walnuts and $15 \mathrm{~g}$ almonds. After adjusting for several characteristics such as smoking or prevalence of certain diseases, those consuming the Mediterranean diet + nuts had a significantly lower risk of having very low plasma BDNF level compared to the low fat diet group. Also, in those diagnosed with depression prior to study enrollment, mean plasma BDNF was higher in Mediterranean diet + nuts group compared to the low fat diet group. BDNF can affect neurotransmitter release, axonal elongation, growth, differentiation, and survival of presynaptic structure [77, 78]. Importantly, BDNF has been demonstrated to prevent or reverse memory loss, cognitive impairment, brain cell degeneration, and cell death in a transgenic mouse model of Alzheimer's disease, as well as aged mouse models $[79,80]$, suggesting that the BDNF enhancement associated with tree nut consumption may have clinical implications for aging and dementia.

A recent study has specifically examined the effects of dietary walnut supplementation on cognitive performance [81]. Young adults were randomly assigned to two conditions using a crossover design, with a 6-week washout between the two conditions: walnuts, then placebo or placebo, then walnuts. Walnuts were consumed in banana bread and the placebo used was banana bread without walnuts. The participants consumed 3 slices of banana bread a day, which, in the walnut-supplemented condition, resulted in about 60 grams of walnuts a day. Data were collected for non-verbal reasoning, verbal reasoning, memory, and mood at baseline, after 8 weeks of walnut supplementation, and after 8 weeks of placebo. There was no effect of short-term walnut supplementation on mood, non-verbal reasoning or memory. However, inferential verbal reasoning significantly increased after walnut supplementation. Thus, it appears that in young and healthy adults, short-term walnut supplementation has a small, but not inconsequential, effect. The short duration of the walnut supplementation could have been a factor, but small improvements due to walnut consumption in healthy, cognitively-intact young adults, could translate into important outcomes in aging populations.

All these reports lend substantial support to the hypothesis that walnuts have the potential to allay agerelated cognitive decline and that the many bioactive 
components of walnuts may have a multitude of beneficial effects on the brain. However, research on the effects of other types of nuts on the brain and behavior is highly limited. Most studies have concentrated on the ability of nut consumption to raise blood antioxidant parameters or reduce signs of inflammation in peripheral tissue or in the blood. However, this is a field of research is open for new scientific study and these data shed light on the potential for these nuts to have an effect on the brain.

\section{Almonds}

Almonds (Prunus, species) are a good source of nutrients like vitamin E, arginine, and fiber [82, 83]. Almonds are also high in fatty acids, particularly LA and oleic fatty acids, which account for over $90 \%$ of the lipids in the almond seed (i.e. kernel) [84]. However, the fatty acid composition varies depending on the cultivar and location in which it was grown [85]. Both the almond pellicle (seed coat) and almond kernel (seed) have many flavonoids, a type of polyphenol with potent antioxidant properties. Extracts of almond pellicle and kernel were determined by high-performance liquid chromatography to contain the flavonoids quercetin, isorhamnetin, quercitrin, kaempferol 3-O-rutinoside, isorhamnetin 3-O-glucoside, and morin [83]. The pellicle of the almond is rich in bioactive compounds. For some preparations, such as blanching, the pellicle is removed, but for others, like roasting, it is not removed. Although the pellicle represents less than 5\% of the total almond weight, it contains $70-100 \%$ of the total phenols present in the almond [86], and has an ORAC of $0.398-0.500 \mathrm{mmol}$ Trolox/g, demonstrating its antioxidant potential [87].

The antioxidant potential of almonds has been demonstrated in humans. A clinical trial [88] was conducted examining the effects of almond consumption on serum parameters of oxidative stress and antioxidants in smokers and non-smokers. Participants were healthy young men (smokers and non-smokers) who consumed either $84 \mathrm{~g}$ of almond powder daily for four weeks or $120 \mathrm{~g}$ of pork daily for four weeks. Fresh boneless pork was chosen because it provides similar energy and protein as almonds. The male smokers whose diet was supplemented with almonds, but not pork, had enhanced serum alpha-tocopherol and plasma antioxidant enzymes, specifically superoxide dismutase and glutathione peroxidase, after 4 weeks of almond supplementation compared to baseline pretreatment concentrations. However, smoking was associated with significantly lower activities of antioxidant enzymes compared to those found in the nonsmokers, regardless of diet. Biomarkers of oxidative stress in smokers who consumed diets containing pork were reduced from baseline to post-diet assessments. Biomarkers of oxidative stress in porkconsuming nonsmokers were not altered. Using the Comet assay, the amount of DNA strand breaks in lymphocytes was determined to be greater in smokers than nonsmokers, but almond consumption reduced DNA strand breaks in smokers compared to pre-intervention levels. Baseline urinary 8-hydroxydeoxyguanosine (8-OHdG) and malondialdehyde (MDA) levels, reflective of oxidative DNA damage and lipid peroxidation, respectively, were higher in smokers compared to nonsmokers, but the almond diet reduced $8-\mathrm{OHdG}$ and MDA levels in smokers. In fact, following almond consumption, urinary MDA in smokers was not significantly different from that of the nonsmokers.

Almonds have also shown potential as a possible adjunctive intervention in Alzheimer's disease [89]. Male rats were orally administered 150,300 , or $600 \mathrm{mg} / \mathrm{kg}$ of a fine paste of almond daily for 7 or 14 days; these doses were selected by conversion from a human dose of 5-6 nuts a day (6 grams). The ability of almond paste consumption to prevent scopolamine-induced amnesia was assessed. Almond paste-fed rats demonstrated significant reversal of some scopolamine-induced cognitive impairment when tested in the elevated-plus maze and in the passive shock avoidance paradigm. The memoryenhancing effects of almond paste may be due to its ability to influence the cholinergic system. Almond paste-fed rats exhibited pro-cholinergic activity, as indicated by reduced brain ACh esterase levels. The cholinergic system is known to play a role in the cognitive dysfunction associated with Alzheimer's disease and aging [90]. These animals also had a reduction in cholesterol and triglyceride levels compared to the rats that did not receive almond paste. This finding is important as cholesterol is thought to be associated with the accumulation of the amyloid plaques associated with Alzheimer's disease [91]. Taken together, these data suggest that the effects of almond consumption on the brain and behavior is a viable avenue for further research, with the goal of identifying foods that help delay the onset of cognitive dysfunction in aging and Alzheimer's disease. 


\section{Pistachios}

It has been demonstrated that consumption of pistachios may improve cholesterol levels [92]. For ten weeks, rats were either fed control diet or one of two different treatment diets, which included pistachios constituting $20 \%$ or $40 \%$ of daily caloric intake. Consumption of pistachio as $20 \%$ of daily caloric intake increased high-density lipoprotein (HDL) levels and decreased total cholesterol (TC)/HDL ratio, compared with those rats that were not consuming pistachios. However, TC, LDL cholesterol, and triglyceride levels were unaffected by pistachio consumption. Compared to the rats consuming control diet, consumption of pistachios as $20 \%$ of daily caloric intake also increased serum paraoxonase and arylesterase activity, which have been shown to inhibit LDL cholesterol oxidation. These results suggest that moderate pistachio consumption may be beneficial for both prevention and treatment of coronary artery disease.

Pistachios are unique nuts, in that they are the only known nut containing anthocyanins, the pigments responsible for the colors of many vegetables and fruits [93]. Various studies in animal models and in humans have suggested that intake of anthocyaninrich foods confers some beneficial effects against age-related neurodegeneration and cognitive decline [94-98]. Anthocyanins are a type of flavonoid and, as indicated in Table 3, pistachios are a rich source of flavonoids, with an average content of about $34 \mathrm{mg} / 100 \mathrm{~g}$ of dry nuts. The level of flavonoids varies in tree nuts as well as in fruits. For example, the level of flavonoids found in blueberries is $117.59 \mathrm{mg} / 100 \mathrm{~g}$, for cranberries is $22.64 \mathrm{mg} / 100 \mathrm{~g}$, and for blackberries is $19.85 / 100 \mathrm{~g}$ [99]. Notably, these fruits have been demonstrated to abrogate age-related cognitive decline in rats $[97,100,101]$, and thus it may be that pistachios have a similar potential if these alterations were at least in part due to the activity of flavonoids in vivo; however, this remains to be tested.

The total content of phenolic compounds in pistachios has been shown to be significantly higher in pellicles than in kernels [93]. Pistachio pellicles and kernels were found to contain gallic acid, catechin, eriodictyol-7-O-glucoside, naringenin-7-Oneohesperidoside, quercetin-3-O-rutinoside and eriodictyol. Genistein-7-O-glucoside, genistein, daidzein and apigenin were found only in pistachio kernels, while epicatechin, quercetin, naringenin, luteolin, kaempferol, cyanidin-3-O-galactoside and cyanidin-
3-O-glucoside were determined to be only in the pistachio pellicles. It appears that in order to optimize the variety of phenolic compounds, one should eat the whole nut, with pellicle intact. The antioxidant activities of pistachio kernels and pellicles were also determined and it was found that the pellicles possessed better antioxidant activity compared to the kernels. The excellent antioxidant activity of pistachio pellicles might be explained, at least in part, by their anthocyanins, which are known antioxidants.

\section{Pecans}

Pecans are a rich source of proanthocyanidins, with an average content of about $494 \mathrm{mg} / 100 \mathrm{~g}$ of dry nuts (Table 3). Although, proanthocyanidin content widely varies among different types of tree nuts (Table 3), proanthocyanidin content from tree nuts is comparable to levels with that of blueberries (cultivated blueberries; $179 \mathrm{mg} / 100 \mathrm{~g}$, wild blueberries; $332 \mathrm{mg} / 100 \mathrm{~g})$, cranberries $(418 \mathrm{mg} / 100 \mathrm{~g})$, and black plums ( $238 \mathrm{mg} / 100 \mathrm{~g})$. Studies have demonstrated that foods high in proanthocyanidins like blueberries, cranberries, and plums have the potential to forestall age-related cognitive declines in rats [98, 100-102] and humans. However, studies specifically examining the effects of pecan consumption on the aging brain either in animals or in humans have not been conducted. Given the bioactive constituents in pecans, this is an avenue of research that is suitable for further study.

Rajaram et al. investigated the ability of a pecansupplemented diet in modifying serum lipids and lipoproteins in men and women with normal to moderately high cholesterol compared to a Step I diet [103]. A Step I diet is a diet recommended by physicians and was developed by the National Cholesterol Education Program to help lower cholesterol. In this cross-over designed study, participants were assigned to follow two different diets, for 4 weeks each: Step I diet and a Step I + pecan diet, which involved reducing all food items in a Step I diet by one fifth, so that total energy was reduced by $20 \%$, and then replacing that portion of the diet with an isoenergetic amount of pecans. Both diets improved the lipid profile, but the consumption of the Step I + pecan diet decreased serum TC, LDL cholesterol, and triglycerides more than the Step I diet alone. The Step I + pecan diet also increased HDL cholesterol. Serum apolipoprotein B decreased and apolipoprotein A1 increased when 
participants consumed the Step I+ pecan diet compared with when they consumed the Step I diet. The favorable alterations that the Step I+ pecan diet had on the serum lipid profile, beyond those that were observed in the Step I diet alone, suggest that pecans may be a healthy recommendation for lowering cholesterol.

In addition to the beneficial effects of the pecan nut itself, researchers have also investigated pecan nut shell extract as a possible dietary supplement. Pecan nut shells are generally a byproduct of pecan processing, but the aqueous extract has sometimes been consumed in tea because of its high antioxidant potential. A recent study sought to determine if consumption of pecan shell extract by rats could prevent the extrapyramidal disorders that developed during typical antipsychotic treatment [104]. These drug-induced motor disorders have been attributed to oxidative damage. Orofacial dyskinesia and catalepsy was induced by acute reserpine or subchronic haloperidol administration either before or after oral treatment with pecan shell aqueous extract, a potential antioxidant. When administered ad libitum for 4 weeks prior to drug-exposure, the pecan shell extract prevented orofacial dyskinesia and catalepsy induced by both drugs. When reserpine and haloperidol were administered before the extract, the animals continued to develop the extrapyramidal symptoms, however, the orofacial parameter was reversed after 7 and 14 days of oral treatment with pecan shell extract. These results suggest the antioxidant potential of pecan shell extract may prevent or reduce some of the oxidative damage induced by these antipsychotic drugs.

Pecan nut shell aqueous extract has also been evaluated for its ability to combat oxidative damage induced by cigarette smoke exposure and behavioral symptoms of smoking withdrawal [105]. Mice were exposed to cigarette smoke for 3 weeks and were orally treated with pecan nut shell extract one week prior to and throughout the 3 weeks of smoke exposure. Pecan nut shell extract prevented oxidative damage, as indicated by reduced brain lipid peroxidation in the mice that received the pecan shell extract during smoke exposure. It also bolstered antioxidant defenses of mice exposed to cigarette smoke, as mice that consumed pecan nut shell extract had higher levels of ascorbic acid in their brains than those mice exposed to smoke who did not. Pecan nut shell extract also reduced the locomotor activity and anxiety symptoms induced by smoking withdrawal. Together, these studies suggest a variety of potential beneficial health effects that pecans and pecan shell extract may have on the cardiovascular system, brain, and behavior. However, one can only speculate as to whether pecan nut or pecan nut shell consumption could mitigate age-related behavioral declines or alterations in neuronal functioning because studies specifically addressing this have not been performed.

\section{A note on dietary supplements}

There are many dietary supplements available that contain various forms of PUFAs, which are found in large amounts in walnuts, but are also found in other tree nuts (Table 2). It is not clear if relying on supplements alone can provide the same benefit as consuming tree nuts. Supplements may not be a suitable replacement for a healthy diet as they cannot replicate all of the nutrients and potential benefits of whole foods. Tree nuts, as discussed, have numerous bioactive constituents like phytochemicals and melatonin, and nutrients like folate and Vitamin E. Thus, in order to fully reproduce the entire array of components that are found in various tree nuts, one would have to rely on numerous supplements beyond those that contain PUFAs. However, previous research has demonstrated that when foods are reduced to individual components, they may not be as effective as the whole food [106, 107].

\section{Conclusions}

Tree nuts may prove to be important nutritional interventions in the prevention and treatment of neurological dysfunction and neurodegeneration. Nuts are nutrient dense and contain a variety of bioactive compounds, including PUFAs and phytochemicals, specifically polyphenols. There is growing evidence that these bioactive constituents have the potential to reduce oxidative stress, inflammation, and cognitive decline associated with aging. Specifically, walnuts have been demonstrated to be able to alter the level of inflammation and oxidative stress in vitro, as well as age-related cognitive decline in rats, and may enhanced cognition in humans as well. Walnuts have the highest level of the omega-3 fatty acid ALA compared to all other edible plants. In fact, the amounts and types of unsaturated fats and phytochemicals vary among 
different types of tree nuts and, thus the effects of tree nuts on health may not be created equal. However, there is a dearth of research examining the direct effects of nut consumption on the brains and behavior of the aging population. This suggests that more research is needed to determine the efficacy of different tree nuts in preventing age-related alterations of the brain and to determine if there are differential effects of tree nuts on the indices associated with the aging brain. Clinical studies supplementing the diets of those individuals with age-related cognitive declines with various amounts of different tree nuts would be the first step in determining if consumption of tree nuts can allay age-related cognitive decline. These studies should also determine the necessary duration of the dietary intervention and if the intervention can be implemented after the occurrence of cognitive declines or only prior to their development. Furthermore, the cellular mechanisms by which nuts may affect neuronal health and functioning in aging are understudied. As is the case with a number of other nutritional substances, it is likely that nuts may exhibit a multiplicity of effects on neural tissue to forestall and reverse motor and cognitive decline during the aging process. While the cure for dementia continues to elude scientists, nutritional intervention with tree nuts, particularly walnuts, may be effective in forestalling the neurological changes associated with aging.

\section{References}

[1] Perry VH. Contribution of systemic inflammation to chronic neurodegeneration. Acta Neuropathol. 2010;120(3):27786.

[2] Romano AD, Serviddio G, de Matthaeis A, Bellanti F, Vendemiale G. Oxidative stress and aging. J Nephrol. 2010;23(Suppl 15):S29-S36.

[3] Douglas PM, Dillin A. Protein homeostasis and aging in neurodegeneration. J Cell Biol. 2010;190(5):719-29.

[4] Joseph J, Cole G, Head E, Ingram D. Nutrition, brain aging, and neurodegeneration. J Neurosci. 2009;29(41): 12795-801.

[5] Floyd RA, Hensley K. Oxidative stress in brain aging. Implications for therapeutics of neurodegenerative diseases. Neurobiol Aging. 2002;23(5):795-807.

[6] Ashford JW, Atwood CS, Blass JP, Bowen RL, Finch CE, Iqbal K, et al. What is aging? What is its role in Alzheimer's disease? What can we do about it? J Alzheimers Dis. 2005;7(3):247-53; discussion 55-62.

[7] Thies W, Bleiler L. Alzheimer's disease facts and figures. Alzheimers Dement. 2011;7(2):208-44.

[8] Yehuda S, Rabinovitz S, Mostofsky DI. Essential fatty acids and the brain: from infancy to aging. Neurobiol Aging. 2005;26(Suppl 1):98-102.
[9] Engelhart MJ, Geerlings MI, Ruitenberg A, van Swieten JC, Hofman A, Witteman JC, et al. Dietary intake of antioxidants and risk of Alzheimer disease. Jama. 2002;287(24): 3223-9.

[10] Commenges D, Scotet V, Renaud S, Jacqmin-Gadda H, Barberger-Gateau P, Dartigues JF. Intake of flavonoids and risk of dementia. Eur J Epidemiol. 2000;16(4):357-63.

[11] Morris MC, Evans DA, Tangney CC, Bienias JL, Wilson RS, Aggarwal NT, et al. Relation of the tocopherol forms to incident Alzheimer disease and to cognitive change. Am J Clin Nutr. 2005;81(2):508-14.

[12] Gu Y, Nieves JW, Stern Y, Luchsinger JA, Scarmeas N. Food combination and Alzheimer disease risk: a protective diet. Arch Neurol. 2010;67(6):699-706.

[13] Salas-Salvado J, Casas-Agustench P, Salas-Huetos A. Cultural and historical aspects of Mediterranean nuts with emphasis on their attributed healthy and nutritional properties. Nutr Metab Cardiovasc Dis. 2011;21(Suppl 1):S1-S6.

[14] United States. Dept. of Agriculture. Human Nutrition Information Service. Dietary Guidelines Advisory Committee., United States. Agricultural Research Service. Report of the Dietary Guidelines Advisory Committee on the dietary guidelines for Americans, 2010 : To the Secretary of Agriculture and the Secretary of Health and Human Services. [Washington, D.C.]: United States Dept. of Agriculture: United States Dept. of Health and Human Services; 2010.

[15] Sabate J, Wien M. Nuts, blood lipids and cardiovascular disease. Asia Pac J Clin Nutr. 2010;19(1):131-6.

[16] Albert CM, Gaziano JM, Willett WC, Manson JE. Nut consumption and decreased risk of sudden cardiac death in the Physicians' Health Study. Arch Intern Med. 2002;162(12): 1382-7.

[17] Fraser GE, Sabate J, Beeson WL, Strahan TM. A possible protective effect of nut consumption on risk of coronary heart disease. The Adventist Health Study. Arch Intern Med. 1992;152(7):1416-24.

[18] Hu FB, Stampfer MJ, Manson JE, Rimm EB, Colditz GA, Rosner BA, et al. Frequent nut consumption and risk of coronary heart disease in women: prospective cohort study. BMJ. 1998;317(7169):1341-5.

[19] Brufau G, Boatella J, Rafecas M. Nuts: Source of energy and macronutrients. Br J Nutr. 2006;96(Suppl 2):S24-8.

[20] Phillips KM, Ruggio DM, Ashraf-Khorassani M. Phytosterol composition of nuts and seeds commonly consumed in the United States. J Agric Food Chem. 2005;53(24):9436-45.

[21] Rajaram S, Sabate J. Nuts, body weight and insulin resistance. Br J Nutr. 2006;96(Suppl 2):S79-S86.

[22] Sacks FM, Katan M. Randomized clinical trials on the effects of dietary fat and carbohydrate on plasma lipoproteins and cardiovascular disease. Am J Med. 2002;113(Suppl 9B):13S24S.

[23] Calder PC. n-3 polyunsaturated fatty acids, inflammation, and inflammatory diseases. Am J Clin Nutr. 2006;83(6 Suppl):1505S-19S.

[24] Haag M. Essential fatty acids and the brain. Can J Psychiatry. 2003;48(3):195-203.

[25] Dyall SC, Michael-Titus AT. Neurological benefits of omega3 fatty acids. Neuromolecular Med. 2008;10(4):219-35. 
[26] Calder PC, Dangour AD, Diekman C, Eilander A, Koletzko B, Meijer GW, et al. Essential fats for future health. Proceedings of the 9th Unilever Nutrition Symposium. Eur J Clin Nutr. 2010;64(Suppl 4):S1-S13.

[27] Cole GM, Ma QL, Frautschy SA. Dietary fatty acids and the aging brain. Nutr Rev. 2010;68(Suppl 2):S102-11.

[28] Willis LM, Bielinski DF, Fisher DR, Matthan NR, Joseph JA. Walnut extract inhibits LPS-induced activation of BV-2 microglia via internalization of TLR4: Possible involvement of phospholipase D2. Inflammation. 2010;33(5):325-33.

[29] Bazan NG. The onset of brain injury and neurodegeneration triggers the synthesis of docosanoid neuroprotective signaling. Cell Mol Neurobiol. 2006;26(4-6):901-13.

[30] Willis LM, Shukitt-Hale B, Joseph JA. Dietary polyunsaturated fatty acids improve cholinergic transmission in the aged brain. Genes Nutr. 2009;4:309-14.

[31] Cole GM, Frautschy SA. DHA may prevent age-related dementia. J Nutr. 2010;140(4):869-74.

[32] Barberger-Gateau P, Letenneur L, Deschamps V, Peres K, Dartigues JF, Renaud S. Fish, meat, and risk of dementia: Cohort study. BMJ. 2002;325(7370):932-3.

[33] Willis LM, Shukitt-Hale B, Cheng V, Joseph JA. Dosedependent effects of walnuts on motor and cognitive function in aged rats. Br J Nutr. 2009;101(8):1140-4.

[34] Solfrizzi V, Colacicco AM, D'Introno A, Capurso C, Torres F, Rizzo C. et al., Dietary intake of unsaturated fatty acids and age-related cognitive decline: A 8.5-year follow-up of the Italian Longitudinal Study on Aging. Neurobiol Aging. 2006;27(11):1694-704.

[35] Simopoulos AP. The importance of the ratio of omega6/omega-3 essential fatty acids. Biomed Pharmacother. 2002;56(8):365-79.

[36] Innis SM. Plasma and red blood cell fatty acid values as indexes of essential fatty acids in the developing organs of infants fed with milk or formulas. J Pediatr. 1992;120(4 Pt 2):S78-S86.

[37] Burdge GC, Wootton SA. Conversion of alpha-linolenic acid to eicosapentaenoic, docosapentaenoic and docosahexaenoic acids in young women. Br J Nutr. 2002;88(4):411-20.

[38] Glaser C, Heinrich J, Koletzko B. Role of FADS1 and FADS2 polymorphisms in polyunsaturated fatty acid metabolism. Metabolism. 2010;59(7):993-9.

[39] USDA. National Nutrient Database for Standard Reference. 2011.

[40] Bolling BW, Chen CY, McKay DL, Blumberg JB. Tree nut phytochemicals: composition, antioxidant capacity, bioactivity, impact factors. A systematic review of almonds, Brazils, cashews, hazelnuts, macadamias, pecans, pine nuts, pistachios and walnuts. Nutr Res Rev. 2011;24(2):244-75.

[41] Vauzour D, Vafeiadou K, Spencer JP. Inhibition of the formation of the neurotoxin 5-S-cysteinyl-dopamine by polyphenols. Biochem Biophys Res Commun. 2007;362(2):340-6.

[42] Devan BD, Goad EH, Petri HL. Dissociation of hippocampal and striatal contributions to spatial navigation in the water maze. Neurobiol Learn Mem. 1996;66:305-23.

[43] Oliveira MGM, Bueno OFA, Pomarico AC, Gugliano EB. Strategies used by hippocampal- and caudate-putamenlesioned rats in a learning task. Neurobiol Learn Mem. 1997;68:32-41.
[44] Vinson JA, Cai Y. Nuts, especially walnuts, have both antioxidant quantity and efficacy and exhibit significant potential health benefits. Food Funct. 2011;3:134-40.

[45] Jurd L. A spectrophotometric method for the detection of odihydroxyl groups in flavonoid compounds. Arch Biochem Biophys. 1956;63(2):376-81.

[46] Blomhoff R, Carlsen MH, Andersen LF, Jacobs DR Jr. Health benefits of nuts: potential role of antioxidants. Br J Nutr. 2006;96(Suppl 2):S52-S60.

[47] Wu X, Beecher GR, Holden JM, Haytowitz DB, Gebhardt SE, Prior RL. Lipophilic and hydrophilic antioxidant capacities of common foods in the United States. J Agric Food Chem. 2004;52(12):4026-37.

[48] Pellegrini N, Serafini M, Salvatore S, Del Rio D, Bianchi M, Brighenti F. Total antioxidant capacity of spices, dried fruits, nuts, pulses, cereals and sweets consumed in Italy assessed by three different in vitro assays. Mol Nutr Food Res. 2006;50(11):1030-8.

[49] Colaric M, Veberic R, Solar A, Hudina M, Stampar F. Phenolic acids, syringaldehyde, and juglone in fruits of different cultivars of Juglans regia L. J Agric Food Chem. 2005;53(16):6390-6.

[50] Vinson JA, Nuts CY. especially walnuts, have both antioxidant quantity and efficacy and exhibit significant potential health benefits. Food Funct. 2012;3(2):134-40.

[51] Feldman EB. The scientific evidence for a beneficial health relationship between walnuts and coronary heart disease. $\mathrm{J}$ Nutr. 2002;132(5):1062S-101S.

[52] Fukuda T, Ito H, Yoshida T. Antioxidative polyphenols from walnuts (Juglans regia L.). Phytochemistry. 2003;63(7): 795-801.

[53] Reiter RJ, Manchester LC, Tan DX. Melatonin in walnuts: influence on levels of melatonin and total antioxidant capacity of blood. Nutrition. 2005;21(9):920-4.

[54] Lahiri DK, Chen DM, Lahiri P, Bondy S, Greig NH. Amyloid, cholinesterase, melatonin, and metals and their roles in aging and neurodegenerative diseases. Ann N Y Acad Sci. 2005;1056:430-49.

[55] Tan DX, Manchester LC, Hardeland R, Lopez-Burillo S, Mayo JC, Sainz RM, et al. Melatonin: a hormone, a tissue factor, an autocoid, a paracoid, and an antioxidant vitamin. J Pineal Res. 2003;34(1):75-8.

[56] Tan DX, Poeggeler B, Reiter RJ, Chen LD, Chen S, Manchester LC, et al. The pineal hormone melatonin inhibits DNA-adduct formation induced by the chemical carcinogen safrole in vivo. Cancer Lett. 1993;70(1-2):65-71.

[57] Zhao G, Etherton TD, Martin KR, West SG, Gillies PJ, Kris-Etherton PM. Dietary alpha-linolenic acid reduces inflammatory and lipid cardiovascular risk factors in hypercholesterolemic men and women. J Nutr. 2004;134(11): 2991-7.

[58] Zhao G, Etherton TD, Martin KR, Gillies PJ, West SG, Kris-Etherton PM. Dietary alpha-linolenic acid inhibits proinflammatory cytokine production by peripheral blood mononuclear cells in hypercholesterolemic subjects. Am J Clin Nutr. 2007;85(2):385-91.

[59] Banel DK, Hu FB. Effects of walnut consumption on blood lipids and other cardiovascular risk factors: a meta-analysis and systematic review. Am J Clin Nutr. 2009;90(1):56-63. 
[60] White WB, Wolfson L, Wakefield DB, Hall CB, Campbell $\mathrm{P}$, Moscufo N, et al. Average daily blood pressure, not office blood pressure, is associated with progression of cerebrovascular disease and cognitive decline in older people. Circulation. 2011;124(21):2312-9.

[61] Roberts RO, Knopman DS, Geda YE, Cha RH, Roger VL, Petersen RC. Coronary heart disease is associated with nonamnestic mild cognitive impairment. Neurobiology of Aging. 2010;31(11):1894-902.

[62] Poulose SM, Bielinski DF, Shukitt-Hale B. Walnut diet reduces accumulation of polyubiquitinated proteins and inflammation in the brain of aged rats. Journal of Nutritional Biochemistry. 2012;60:1084-93.

[63] Haider S, Batool Z, Tabassum S, Perveen T, Saleem S, Naqvi F, et al. Effects of walnuts (Juglans regia) on learning and memory functions. Plant Foods Hum Nutr. 2011;66(4): 335-40.

[64] Haider S, Khaliq S, Ahmed SP, Haleem DJ. Long-term tryptophan administration enhances cognitive performance and increases 5HT metabolism in the hippocampus of female rats. Amino Acids. 2006;31(4):421-5.

[65] Haider S, Khaliq S, Haleem DJ. Enhanced serotonergic neurotransmission in the hippocampus following tryptophan administration improves learning acquisition and memory consolidation in rats. Pharmacol Rep. 2007;59(1):53-7.

[66] Heuser IJ, Gotthardt U, Schweiger U, Schmider J, Lammers $\mathrm{CH}$, Dettling M, et al. Age-associated changes of pituitaryadrenocortical hormone regulation in humans: importance of gender. Neurobiol Aging. 1994;15(2):227-31.

[67] Otte C, Hart S, Neylan TC, Marmar CR, Yaffe K, Mohr DC. A meta-analysis of cortisol response to challenge in human aging: importance of gender. Psychoneuroendocrinology. 2005;30(1):80-91.

[68] Nemeroff CB, Owens MJ. Pharmacologic differences among the SSRIs: focus on monoamine transporters and the HPA axis. CNS Spectr. 2004;9(6 Suppl 4):23-31.

[69] Glenner GG. Alzheimer's disease. The commonest form of amyloidosis. Arch Pathol Lab Med. 1983;107(6):281-2.

[70] Masters CL, Multhaup G, Simms G, Pottgiesser J, Martins RN, Beyreuther K, Neuronal. origin of a cerebral amyloid: neurofibrillary tangles of Alzheimer's disease contain the same protein as the amyloid of plaque cores and blood vessels. EMBO J. 1985;4(11):2757-63.

[71] Bonda DJ, Wang X, Perry G, Nunomura A, Tabaton M, Zhu $\mathrm{X}$, et al. Oxidative stress in Alzheimer disease: a possibility for prevention. Neuropharmacology. 2010;59(4-5):290-4 .

[72] Harris ME, Hensley K, Butterfield DA, Leedle RA, Carney JM. Direct evidence of oxidative injury produced by the Alzheimer's beta-amyloid peptide (1-40) in cultured hippocampal neurons. Exp Neurol. 1995;131(2):193-202.

[73] Muthaiyah B, Essa MM, Chauhan V, Chauhan A. Protective effects of walnut extract against amyloid beta peptideinduced cell death and oxidative stress in PC12 cells. Neurochem Res. 2011;36(11):2096-103.

[74] Lorenzo A, Yankner BA. Beta-amyloid neurotoxicity requires fibril formation and is inhibited by congo red. Proc Natl Acad Sci U S A. 1994;91(25):12243-7.

[75] Chauhan N, Wang KC, Wegiel J, Malik MN. Walnut extract inhibits the fibrillization of amyloid beta-protein, and also defibrillizes its preformed fibrils. Curr Alzheimer Res. 2004;1(3):183-8.

[76] Sanchez-Villegas A, Galbete C, Martinez-Gonzalez MA, Martinez JA, Razquin C, Salas-Salvado J, et al. The effect of the Mediterranean diet on plasma brain-derived neurotrophic factor (BDNF) levels: the PREDIMED-NAVARRA randomized trial. Nutr Neurosci. 2011;14(5):195-201.

[77] Ventimiglia R, Mather PE, Jones BE, Lindsay RM. The neurotrophins BDNF, NT-3 and NT-4/5 promote survival and morphological and biochemical differentiation of striatal neurons in vitro. Eur J Neurosci. 1995;7(2):213-22.

[78] Wang T, Xie K, Lu B. Neurotrophins promote maturation of developing neuromuscular synapses. J Neurosci. 1995;15(7 Pt 1):4796-805.

[79] Nagahara AH, Merrill DA, Coppola G, Tsukada S, Schroeder BE, Shaked GM, et al. Neuroprotective effects of brainderived neurotrophic factor in rodent and primate models of Alzheimer's disease. Nat Med. 2009;15(3):331-7.

[80] Peng S, Wuu J, Mufson EJ, Fahnestock M. Precursor form of brain-derived neurotrophic factor and mature brain-derived neurotrophic factor are decreased in the pre-clinical stages of Alzheimer's disease. J Neurochem. 2005;93(6):1412-21.

[81] Pribis P, Bailey RN, Russell AA, Kilsby MA, Hernandez M, Craig WJ, et al. Effects of walnut consumption on cognitive performance in young adults. Br J Nutr. 2011;19:1-9.

[82] Song Y, Wang W, Cui W, Zhang X, Zhang W, Xiang Q, et al. A subchronic oral toxicity study of almond skins in rats. Food Chem Toxicol. 2010;48(1):373-6.

[83] Wijeratne SS, Abou-Zaid MM, Shahidi F. Antioxidant polyphenols in almond and its coproducts. J Agric Food Chem. 2006;54(2):312-8.

[84] Sathe SK. Solubilization, Electrophoretic Characterization and Invitro Digestibility of Almond (Prunus-Amygdalus) Proteins. J Food Biochem. 1993;16(4):249-64.

[85] Sathe SK, Seeram NP, Kshirsagar HH, Heber D, Lapsley KA. Fatty acid composition of California grown almonds. J Food Sci. 2008;73(9):C607-14.

[86] Milbury PE, Chen CY, Dolnikowski GG, Blumberg JB. Determination of flavonoids and phenolics and their distribution in almonds. J Agric Food Chem. 2006;54(14): 5027-33.

[87] Monagas M, Garrido I, Lebron-Aguilar R, Bartolome B, Gomez-Cordoves C, Almond. (Prunus dulcis (Mill.) D.A. Webb) skins as a potential source of bioactive polyphenols. J Agric Food Chem. 2007;55(21):8498-507.

[88] Li N, Jia X, Chen CY, Blumberg JB, Song Y, Zhang W, et al. Almond consumption reduces oxidative DNA damage and lipid peroxidation in male smokers. J Nutr. 2007;137(12): 2717-22.

[89] Kulkarni KS, Kasture SB, Mengi SA. Efficacy study of Prunus amygdalus (almond) nuts in scopolamine-induced amnesia in rats. Indian J Pharmacol. 2010;42(3):168-73.

[90] Ellis JM. Cholinesterase inhibitors in the treatment of dementia. J Am Osteopath Assoc. 2005;105(3):145-58.

[91] Refolo LM, Malester B, LaFrancois J, Bryant-Thomas T, Wang R, Tint GS, et al. Hypercholesterolemia accelerates the Alzheimer's amyloid pathology in a transgenic mouse model. Neurobiol Dis. 2000;7(4):321-31. 
[92] Aksoy N, Aksoy M, Bagci C, Gergerlioglu HS, Celik H, Herken E, et al. Pistachio intake increases high density lipoprotein levels and inhibits low-density lipoprotein oxidation in rats. Tohoku J Exp Med. 2007;212(1):43-8.

[93] Tomaino A, Martorana M, Arcoraci T, Monteleone D, Giovinazzo C, Saija A. Antioxidant activity and phenolic profile of pistachio (Pistacia vera L., variety Bronte) seeds and skins. Biochimie. 2010;92(9):1115-22.

[94] Krikorian R, Shidler MD, Nash TA, Kalt W, VinqvistTymchuk MR, Shukitt-Hale B, et al. Blueberry supplementation improves memory in older adults. J Agric Food Chem. 2010;58(7):3996-4000.

[95] Shukitt-Hale B, Carey A, Simon L, Mark DA, Joseph JA. Effects of Concord grape juice on cognitive and motor deficits in aging. Nutrition. 2006;22(3):295-302.

[96] Krikorian R, Nash TA, Shidler MD, Shukitt-Hale B, Joseph JA. Concord grape juice supplementation improves memory function in older adults. British Journal of Nutrition. 2010;23:1-5.

[97] Shukitt-Hale B, Cheng V, Joseph JA. Effects of blackberries on motor and cognitive function in aged rats. Nutr Neurosci. 2009;12(3): 135-40

[98] Shukitt-Hale B, Kalt W, Carey AN, Vinqvist-Tymchuk M, McDonald J, Joseph JA. Plum juice, but not dried plum powder, is effective in mitigating cognitive deficits in aged rats. Nutrition. 2009;25(5):567-73.

[99] USDA. USDA Database for the Flavonoid Content of Selected Foods. 2011.

[100] Joseph JA, Shukitt-Hale B, Casadesus G. Reversing the deleterious effects of aging on neuronal communication and behavior: Beneficial properties of fruit polyphenolic compounds. Am J Clin Nutr. 2005;81(1 Suppl):313S-6S .

[101] Shukitt-Hale B, Carey AN, Jenkins D, Rabin BM, Joseph JA. Beneficial effects of fruit extracts on neuronal function and behavior in a rodent model of accelerated aging. Neurobiol Aging. 2007;28(8):1187-94.

[102] Galli RL, Shukitt-Hale B, Youdim KA, Joseph JA. Fruit polyphenolics and brain aging: nutritional interventions targeting age-related neuronal and behavioral deficits. Ann N Y Acad Sci. 959:128-32.

[103] Rajaram S, Burke K, Connell B, Myint T, Sabate J. A monounsaturated fatty acid-rich pecan-enriched diet favorably alters the serum lipid profile of healthy men and women. J Nutr. 2001;131(9):2275-9.

[104] Trevizol F, Benvegnu DM, Barcelos RC, Pase CS, Segat HJ, Dias VT, et al. Comparative study between two animal models of extrapyramidal movement disorders: prevention and reversion by pecan nut shell aqueous extract. Behav Brain Res. 2011;221(1):13-8.

[105] Reckziegel P, Boufleur N, Barcelos RC, Benvegnu DM, Pase CS, Muller LG, et al. Oxidative stress and anxiety-like symptoms related to withdrawal of passive cigarette smoke in mice: beneficial effects of pecan nut shells extract, a byproduct of the nut industry. Ecotoxicol Environ Saf. 2011 Sep;74(6):1770-8.

[106] Liu RH. Health benefits of fruit and vegetables are from additive and synergistic combinations of phytochemicals. Am J Clin Nutr. 2003;78(3 Suppl):517S-20S

[107] Carey AN, Fisher DR, Joseph JA, Shukitt-Hale B. The ability of walnut extract and fatty acids to protect against the deleterious effects of oxidative stress and inflammation in hippocampal cells. Nutr Neurosci. in press.

[108] USDA. Fruit and Tree Nuts Situation and Outlook Yearbook 2011. 2011.

[109] Maguire LS, O'Sullivan SM, Galvin K, O'Connor TP, O'Brien NM. Fatty acid profile, tocopherol, squalene and phytosterol content of walnuts, almonds, peanuts, hazelnuts and the macadamia nut. Int J Food Sci Nutr. 2004;55(3): 171-8.

[110] Ryan E, Galvin K, O'Connor TP, Maguire AR, O'Brien NM. Fatty acid profile, tocopherol, squalene and phytosterol content of brazil, pecan, pine, pistachio and cashew nuts. Int J Food Sci Nutr. 2006;57(3-4):219-28. 